\title{
Wound Complications in Preoperatively Irradiated Soft-Tissue Sarcomas of the Extremities
}

\author{
Lewis A. Rosenberg, MD, PhD*, Robert J. Esther, MD $^{\dagger}$, Kamil Erfanian, MD ${ }^{\ddagger}$, Rebecca \\ Green, MSW ${ }^{\star}$, Hong Jin Kim, MD ${ }^{\ddagger}$, Raeshell Sweeting, MD ${ }^{\ddagger}$, and Joel E. Tepper, MD \\ *Department of Radiation Oncology, University of North Carolina, Chapel Hill, North Carolina \\ tDepartment of Orthopedics, University of North Carolina, Chapel Hill, North Carolina \\ ‡Department of Surgery, University of North Carolina, Chapel Hill, North Carolina
}

\begin{abstract}
Purpose-To determine whether the involvement of plastic surgery and the use of vascularized tissue flaps reduces the frequency of major wound complications after radiation therapy for softtissue sarcomas (STS) of the extremities.
\end{abstract}

\begin{abstract}
Methods and Materials-This retrospective study evaluated patients with STS of the extremities who underwent radiation therapy before surgery. Major complications were defined as secondary operations with anesthesia, seroma/hematoma aspirations, readmission for wound complications, or persistent deep packing.
\end{abstract}

\begin{abstract}
Results-Between 1996 and 2010, 73 patients with extremity STS were preoperatively irradiated. Major wound complications occurred in 32\% and secondary operations in 16\% of patients. Plastic surgery closed $63 \%$ of the wounds, and vascularized tissue flaps were used in $22 \%$ of closures. When plastic surgery performed closure the frequency of secondary operations trended lower ( $11 \%$ vs $26 \% ; P=.093$ ), but the frequency of major wound complications was not different ( $28 \%$ vs $38 \% ; P=.43$ ). The use of a vascularized tissue flap seemed to have no effect on the frequency of complications. The occurrence of a major wound complication did not affect disease recurrence or survival. For all patients, 3-year local control was 94\%, and overall survival was $72 \%$.
\end{abstract}

Conclusions-The rates of wound complications and secondary operations in this study were very similar to previously published results. We were not able to demonstrate a significant relationship between the involvement of plastic surgery and the rate of wound complications, although there was a trend toward reduced secondary operations when plastic surgery was involved in the initial operation. Wound complications were manageable and did not compromise outcomes. 


\section{Introduction}

Historically, without the use of additional therapy, amputations or large compartmental excisions were required to achieve acceptable local control rates for soft tissue sarcomas (STS) of the extremities (1). A randomized controlled trial in 1982 demonstrated that the addition of radiation therapy (RT) to limb-sparing surgery achieved acceptable local control rates when compared with amputation, with no difference in survival (2). Other randomized controlled trials have confirmed the value of RT as an integral part of limb-sparing treatment for high-grade or large STS $(3,4)$.

The timing of RT with respect to surgery is controversial. O'Sullivan et al randomized patients with STS to preoperative or postoperative RT. Results ultimately demonstrated no difference in local control or overall survival, although an early report suggested a survival benefit with preoperative radiation $(5,6)$. Although oncologic outcomes seem independent of radiation timing, several studies have shown significant differences in the rate of complications: major wound complications are more common with preoperative RT, and late complications may be more common with postoperative RT (5-9).

Our institutional preference has been to irradiate preoperatively STS that are high grade, large, or predicted to be difficult to resect with negative margins, such as those abutting major vessels or nerves. In an attempt to reduce the risk of wound complications, the plastic surgery service is routinely involved in choosing the wound closure approach and frequently performs closure, often with vascularized tissue flaps. We hypothesized that the use of vascularized tissue flaps and/or closure by the plastic surgery service would be associated with a lower rate of major wound complications. We therefore evaluated the frequency of major wound complications at our institution in patients with STS of the extremities who were preoperatively irradiated and determined which patient and tumor characteristics predict for such complications.

\section{Methods and Materials}

With the approval of our institutional review board (IRB \#11-0019), a retrospective study was undertaken to evaluate wound complications in patients with STS of the extremities who underwent preoperative RT. Patients eligible for this study were older than 18 years, had at least 4 months of follow-up from the time of surgery, underwent primary surgery at our institution, and were treated with preoperative RT either at our or another institution. Rhabdomyosarcoma, Ewing sarcoma, and dermatifibrosarcoma protuberans were excluded from this study because these malignancies differ in therapy and natural history compared with standard adult extremity STS. Several existing patient databases at our institution were searched for eligible patients, and data were collected using electronic and print medical records, patient phone calls, and the social security death index.

Upper extremity sarcomas were defined as those between the fingers and the medial border of the scapula; lower extremity sarcomas were those between the toes and the upper border of the iliac crest (excluding internal pelvic tumors). Any discordance between the pathology (including grade) of the biopsy and the operative pathology was resolved by favoring the 
biopsy, because this tissue was unirradiated. If the biopsy yielded incomplete information, the pathology report from the surgery was used. Pathologists have used multiple approaches over the years of this study for determining sarcoma grade at our institution. For the purposes of this article, any pathology labeled "high grade," "intermediate grade," "grade 3," or "grade 2" was considered "high grade"; and any pathology labeled "low grade" or "grade 1" was considered "low grade."

Wound closure approaches were placed into the following categories: primary closure (simple reapproximation of tissues), healing by secondary intention, local tissue rearrangement (ie, advancement flap), regional flap (ie, pedicled flap), and free flap. The term "unirradiated tissue flap" includes both regional and free flaps.

The definition of "major wound complication" was by design identical to that used in the O’Sullivan (2002) trial (5), reproduced here: "[M]ajor wound complication [was] defined as a secondary operation under general or regional anesthesia for wound repair (debridement, operative drainage, and secondary wound closure including rotationplasty, free flaps, or skin grafts), or wound management without secondary operation. Wound management included an invasive procedure without general or regional anaesthesia (mainly aspiration of seroma), readmission for wound care such as intravenous antibiotics, or persistent deep packing for 120 days or longer." By this definition, in clinic debridements or seroma/hematoma aspirations were considered major wound complications.

Survival curves and statistics were generated using the Kaplan-Meier method. Univariate analyses for categoric variables and continuous variables were performed with $\chi^{2}$ tests and regression analyses, respectively. Multivariate analyses were performed using forward selection and considered only those variables with a $P$ value of $<.1$ by univariate analyses. All $P$ values are 2-tailed. Median follow-up was calculated using the method of Schemper and Smith (10).

\section{Results}

Seventy-three patients with STS of the extremities were identified who were preoperatively irradiated and underwent resection with curative intent at our institution between 1996 and 2010. Patient and tumor characteristics are listed in either Table 1 or as part of the univariate analysis in Table 2. Other pertinent data are as follows: median patient age was 56 years (range, 19-85 years). Average tumor size was $12.2 \mathrm{~cm}, 62 \%(\mathrm{n}=45)$ of tumors were at least $10 \mathrm{~cm}$ in largest dimension, and $21 \%(\mathrm{n}=15)$ were at least $20 \mathrm{~cm}$ in largest dimension.

Fifty-four patients (74\%) received radiation at our institution, and $19(26 \%)$ were irradiated elsewhere before undergoing resection at our institution. Median radiation dose was 5000 cGy and ranged from 4320 cGy-5400 cGy. Fraction size was 200 cGy for 19 patients, 180 cGy for 42 patients, and mixed or unknown for 12 patients. Six patients were treated with an external beam postoperative RT boost for positive margins (dose range, 2000-3000 cGy), 3 were treated with postoperative brachytherapy with catheters placed at the time of surgery (all received $1500 \mathrm{cGy}$ at $0.5 \mathrm{~cm}$ from plane of the catheters), and 4 had intraoperative external-beam RT to $1000 \mathrm{cGy}$. Chemotherapy was administered to $18 \%$ of patients $(\mathrm{n}=13)$ 
(12 before RT and 1 after surgery); all received adriamycin and ifosfamide. At 3 years, local control was $94 \%$, overall survival was $72 \%$, and disease-free survival was $61 \%$ (Fig. 1). Median follow-up time was 3.5 years.

All patients underwent a gross total resection, and $18 \%(\mathrm{n}=13)$ had positive margins. Plastic surgery closed the wound in $63 \%(n=46)$ of cases. There was shift in the general approach to the inclusion of the plastic surgery service during the time of this study: before October 2001, 2 of 19 cases (11\%) involved plastic surgery, and after that date 44 of 54 cases $(81 \%)$ involved plastic surgery. Wound closure was achieved as follows: $51 \%(\mathrm{n}=37)$ healing by primary intention; $5 \%(\mathrm{n}=4)$ healing by secondary intention; $22 \%(\mathrm{n}=16)$ local tissue rearrangement; $19 \%(\mathrm{n}=14)$ regional flap reconstruction; and 3\% $(\mathrm{n}=2)$ free flap reconstruction. Split-thickness skin grafts were used in $16 \%$ of patients $(n=12)$.

The overall frequency of major wound complications was 32\% and of secondary operations for wound closure was $16 \%$. Details of wound complications are listed in Table 3.

Univariate analysis was undertaken to evaluate variables that may have influenced the frequency of wound complications and secondary operations (Table 2). The frequency of major complications when wounds were closed by plastic surgery was $28 \%$, compared with $37 \%$ when plastic surgery was not involved; this difference was not statistically significant $(P=.44)$. The absolute frequency of secondary operations was lower when plastic surgery was involved (11\% vs $26 \%)$, although this difference only approached statistical significance $\left(P=.093, \chi^{2}\right.$ test). As shown in Table 2, the use of an unirradiated tissue flap did not influence the frequency of wound complications or secondary operations. To determine whether there was a bias for larger tumors to undergo more-aggressive closure with plastic surgery or vascularized tissue flaps, we determined the average size of tumors with and without these interventions. The average size of a tumor when plastic surgery was involved was $12.7 \mathrm{~cm}$, compared with $11.4 \mathrm{~cm}$ without $(P=.46$, unpaired $t$ test), and the average size of a tumor closed with an unirradiated flap was $14.2 \mathrm{~cm}$, compared with 11.7 $\mathrm{cm}$ without $(P=.22$, unpaired $t$ test $)$.

The relationships of patient and tumor characteristics with major wound complications and secondary operations were further evaluated with multivariate analysis. For the outcome of secondary operation, only gender, institution performing RT, and grade were identified as significant factors (with a higher likelihood of secondary operations in women, those patients not irradiated at our institution, and low-grade tumors, respectively; Table 4). According to multivariate analysis, no variables were significantly associated with major wound complications.

All major wound complications resolved with intervention, and no deaths or permanent disability resulted. In patients with major wound complications vs those without, at 3 years overall survival was $64 \%$ vs $77 \%$, disease-free survival was $53 \%$ vs $66 \%$, and local control was $84 \%$ vs $100 \%$ (Fig. 2). None of these differences approached statistical significance (log-rank test). 


\section{Discussion}

Radiation therapy is well established as a standard component of limb-sparing therapy for large or high-grade STS of the extremities, but the timing of RT is controversial. O'Sullivan et al (5) provided the best assessment of this issue through a randomized trial that assigned patients with STS undergoing limb-sparing surgery to either preoperative or postoperative RT. The initial publication of this study showed no difference in local, regional, or distant control but did find a higher rate of wound complications with preoperative RT, as well as a significant overall survival benefit with preoperative RT (5). With longer follow-up, this overall survival difference was not confirmed, and the authors concluded that there is no difference in oncologic outcomes according to the timing of RT (6). The same trial evaluated late effects of radiation and found trends toward higher morbidity in patients who received postoperative RT (9). Ultimately the O'Sullivan trial showed that the timing of RT did not affect oncologic outcomes but that preoperative RT possibly led to fewer late effects at the cost of more frequent acute wound complications.

Our institutional preference is to preoperatively irradiate STS of the extremities. In recent years our policy has been for the plastic surgery service to lead the discussions that determine the wound closure approach for each patient. Because the ultimate decision as to wound closure approach is frequently made when the wound is assessed at the time of surgery, the plastic surgery service frequently performs closure regardless of the approach. We hypothesized that the frequent involvement of the plastic surgery service and the use of vascularized tissue flaps would yield a lower rate of wound complications. This retrospective study was designed to evaluate complications in patients with extremity STS who underwent resection at our institution. To facilitate comparisons, the definitions of "major wound complication" and "secondary operation" were identical to those used by O'Sullivan et al (5).

We found rates of complications that were quite similar to other studies reporting outcomes of preoperatively irradiated extremity STS. O'Sullivan et al (5) reported a frequency of $35 \%$ for major wound complications, compared with $32 \%$ in this study. A potential criticism of this definition of "major wound complication" is that it includes events that may not include significant morbidity, such as seroma aspirations performed in clinic. As such, secondary operations were assessed separately, because this event is more reproducible and is universally agreed upon as a true clinically significant major complication. We reported a frequency of $16 \%$ for secondary operations, an identical result to that of O'Sullivan et al (5). Other studies have found similar results. Cannon et al (2006) (8) retrospectively evaluated the experience at MD Anderson Cancer Center with preoperatively irradiated STS and found an acute wound complication rate of $34 \%$ for 269 patients with preoperatively irradiated STS. Another smaller study of 48 patients found a wound complication rate of $41 \%$ and a secondary operation rate of $18 \%$ with a similar definition of wound complication (11). Temple et al (2007) (12) reported a favorable $11 \%$ rate of secondary operation, but this study included only upper-extremity tumors, which were typically smaller, and patients only received 30 Gy in 10 fractions preoperatively. 
The present study did not identify any statistically significant association between closure by the plastic surgery service or the use of vascularized tissue flaps and the rate of major wound complications. It should be noted that with the use of plastic surgery, the secondary operation rate was a manageable $11 \%$ (vs $26 \%$ without plastic surgery), although this difference only trended toward significance. Other studies have also failed to find an association between the use of plastic surgery or tissue flaps and the frequency of wound complications $(8,13)$. Interestingly, 1 study reported a trend toward higher complications when plastic surgery closed (8). This seemingly counterintuitive finding may be confounded by a selection bias whereby larger and more invasive tumors are more often closed by plastic surgery with or without the use of a tissue flap. We found that larger tumors did tend to be closed more often by plastic surgery and more often utilized a vascularized tissue flap, but these findings were not statistically significant. Our univariate analysis also found a higher rate of secondary operations in lower-extremity tumors, women, low-grade tumors, and those not receiving radiation at our institution; the latter 3 findings were also statistically significant with multivariate analysis. The finding that lower-extremity tumors tend to have a higher complication rate has been previously shown, but the other associations have not been replicated (5). It is unknown why patients irradiated at our institution had a lower rate of secondary operations, although one might postulate that there were differences in irradiation technique and/or patient management responsible for this finding.

A major limitation of this study is that it is retrospective, so there are likely many unknown biases affecting the data. Additionally, the data set is relatively small, with only 73 patients and few reported events (23 wound complications and 12 secondary operations). The statistical power is therefore limited, raising the possibility that this study has failed to identify important correlations. For example, the $15 \%$ absolute reduction in secondary operations with the involvement of plastic surgery may be clinically significant, but this study was underpowered to confirm this association. A final weakness of this study is that we do not report data on late effects and functional outcomes, which are important considerations for the timing of RT. Although our data on wound complications are robust, our chart review quickly revealed that data concerning late effects are less consistently recorded in the medical records, and we lacked confidence in these data.

In conclusion, this study demonstrates that the frequency of acute wound complications was not significantly diminished with routine use of plastic surgery, but the frequency of secondary operations may be less when plastic surgery closes the initial resection. Because the complication rate was not definitively altered by these interventions, it seems that the risk of complication may be more dependent on the nature of surgery required in these often large and difficult to resect tumors, rather than the type of closure. Although acute wound complications are more frequent with preoperative RT, the complications are manageable and do not compromise outcomes. Furthermore, preoperative RT, when compared with postoperative, typically involves a lower dose of radiation and a smaller field and may yield fewer late complications. Our institutional approach will likely continue to include preoperative RT for limb-sparing surgery for STS. 


\section{References}

1. Shiu MH, Castro EB, Hajdu SI, et al. Surgical treatment of 297 soft tissue sarcomas of the lower extremity. Ann Surg. 1975; 182:597-602. [PubMed: 1190864]

2. Rosenberg SA, Tepper J, Glatstein E, et al. The treatment of soft-tissue sarcomas of the extremities: prospective randomized evaluations of (1) limb-sparing surgery plus radiation therapy compared with amputation and (2) the role of adjuvant chemotherapy. Ann Surg. 1982; 196:305-315. [PubMed: 7114936]

3. Pisters PW, Harrison LB, Leung DH, et al. Long-term results of a prospective randomized trial of adjuvant brachytherapy in soft tissue sarcoma. J Clin Oncol. 1996; 14:859-868. [PubMed: 8622034]

4. Yang JC, Chang AE, Baker AR, et al. Randomized prospective study of the benefit of adjuvant radiation therapy in the treatment of soft tissue sarcomas of the extremity. J Clin Oncol. 1998; 16:197-203. [PubMed: 9440743]

5. O'Sullivan B, Davis AM, Turcotte R, et al. Preoperative versus postoperative radiotherapy in softtissue sarcoma of the limbs: a randomised trial. Lancet. 2002; 359:2235-2241. [PubMed: 12103287]

6. O'Sullivan B, Davis AM, Turcotte R, et al. Five-year results of a randomized phase III trial of preoperative versus post-operative radiotherapy in extremity soft tissue sarcoma. J Clin Oncol. 2004:22.

7. Al-Absi E, Farrokhyar F, Sharma R, et al. A systematic review and meta-analysis of oncologic outcomes of pre-versus postoperative radiation in localized resectable soft-tissue sarcoma. Ann Surg Oncol. 2010; 17:1367-1374. [PubMed: 20217260]

8. Cannon CP, Ballo MT, Zagars GK, et al. Complications of combined modality treatment of primary lower extremity soft-tissue sarcomas. Cancer. 2006; 107:2455-2461. [PubMed: 17036354]

9. Davis AM, O’Sullivan B, Turcotte R, et al. Late radiation morbidity following randomization to preoperative versus postoperative radio-therapy in extremity soft tissue sarcoma. Radiother Oncol. 2005; 75:48-53. [PubMed: 15948265]

10. Schemper M, Smith TL. A note on quantifying follow-up in studies of failure time. Control Clin Trials. 1996; 17:343-346. [PubMed: 8889347]

11. Hui AC, Ngan SY, Wong K, et al. Preoperative radiotherapy for soft tissue sarcoma: the Peter MacCallum Cancer Centre experience. Eur J Surg Oncol. 2006; 32:1159-1164. [PubMed: 16765559]

12. Temple CL, Ross DC, Magi E, et al. Preoperative chemoradiation and flap reconstruction provide high local control and low wound complication rates for patients undergoing limb salvage surgery for upper extremity tumors. J Surg Oncol. 2007; 95:135-141. [PubMed: 17262730]

13. Kunisada T, Ngan SY, Powell G, et al. Wound complications following pre-operative radiotherapy for soft tissue sarcoma. Eur J Surg Oncol. 2002; 28:75-79. [PubMed: 11869019] 


\section{Summary}

Wound complications are a significant concern when soft-tissue sarcomas of the extremities are irradiated before resection. This retrospective study evaluated such patients and sought to determine what factors influence the occurrence of wound complications. We found that the frequency of wound complications remains concerning even with aggressive and careful interventions, but a trend suggested that complications are less frequent when plastic surgery performs closure. 


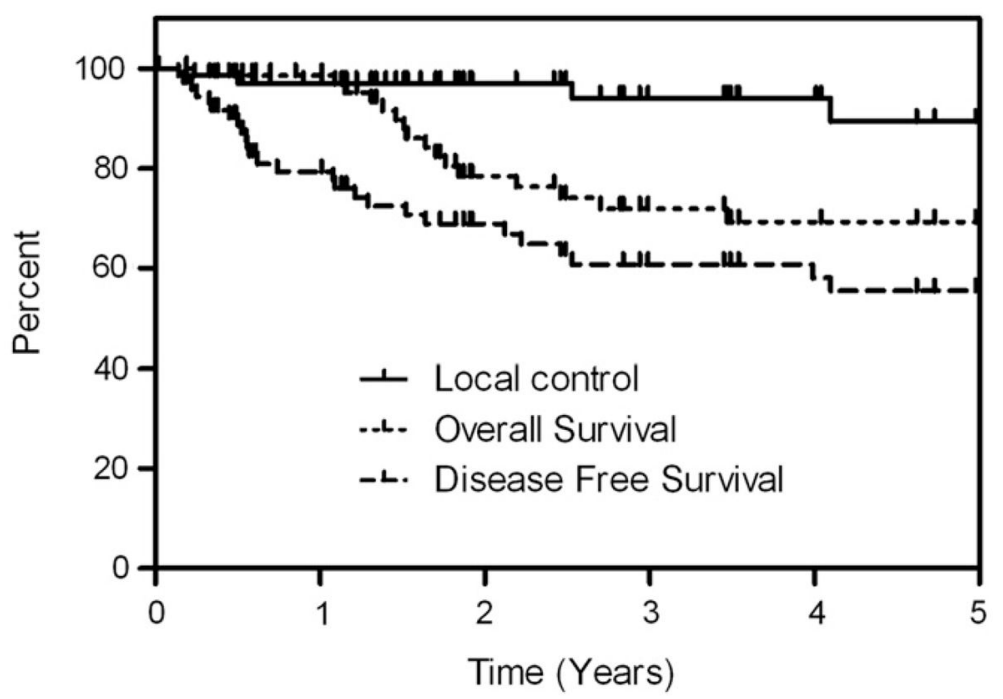

Fig. 1.

Local control, overall survival, and disease-free survival for all patients as determined by the Kaplan-Meier method. Tick marks represent censored patients. 

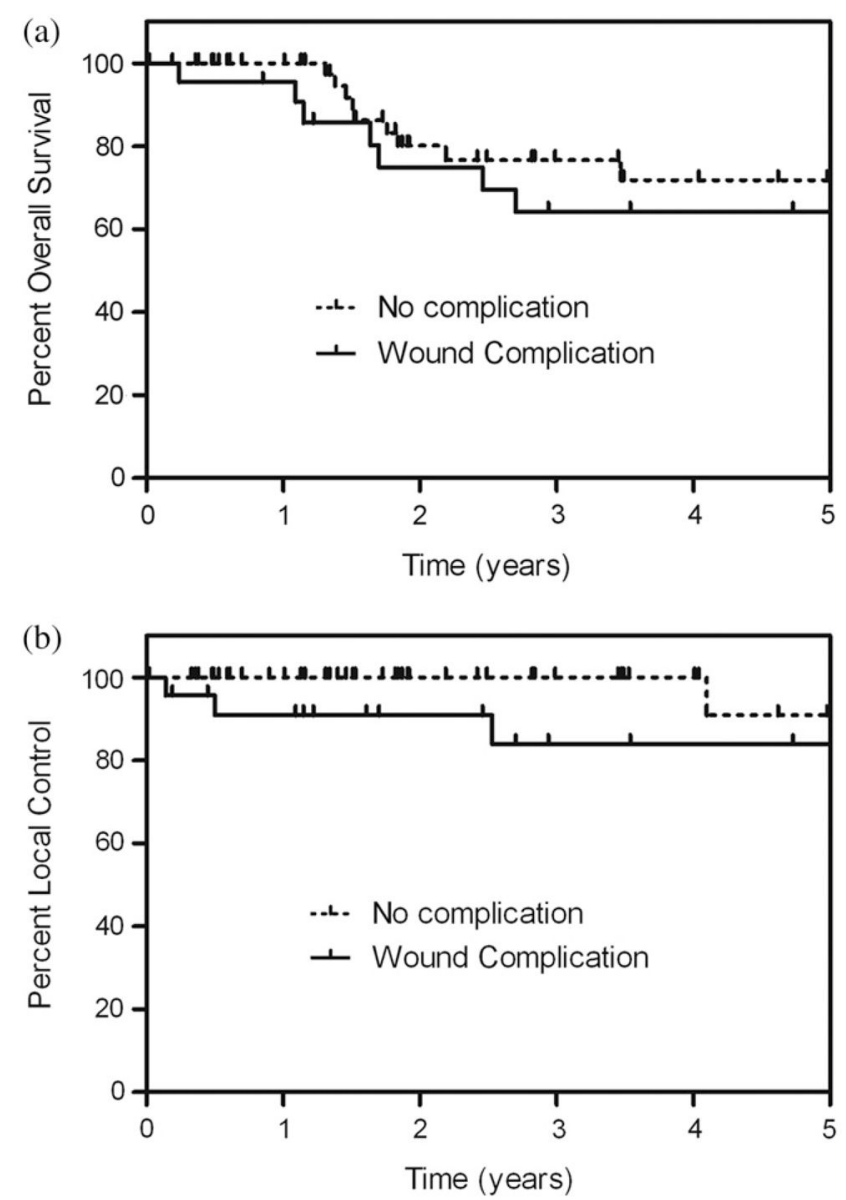

Fig. 2.

Overall survival (a) and local control (b) for patients with and without major wound complications. Tick marks represent censored patients. 


\section{Table 1}

\section{Patient and tumor characteristics}

\begin{tabular}{|c|c|c|}
\hline Characteristic & $\mathbf{n}$ & $\%$ \\
\hline \multicolumn{3}{|l|}{ Ethnicity } \\
\hline African American & 21 & 29 \\
\hline Caucasian/Hispanic & 51 & 70 \\
\hline Unknown & 1 & 1 \\
\hline \multicolumn{3}{|l|}{ Pathology } \\
\hline Pleomorphic & 13 & 18 \\
\hline Myxofibrosarcoma & 9 & 12 \\
\hline Liposarcoma & 8 & 11 \\
\hline Myxoid liposarcoma & 8 & 11 \\
\hline Pleomorphic liposarcoma & 7 & 10 \\
\hline MPNST & 4 & 5 \\
\hline Synovial & 3 & 4 \\
\hline Other ${ }^{*}$ & 21 & 29 \\
\hline \multicolumn{3}{|l|}{ Location } \\
\hline Right upper extremity & 13 & 18 \\
\hline Left upper extremity & 11 & 15 \\
\hline Right lower extremity & 22 & 30 \\
\hline Left lower extremity & 27 & 37 \\
\hline \multicolumn{3}{|l|}{ Clinical tumor stage } \\
\hline T1a & 5 & 7 \\
\hline $\mathrm{T} 1 \mathrm{~b}$ & 6 & 8 \\
\hline $\mathrm{T} 2 \mathrm{a}$ & 4 & 5 \\
\hline $\mathrm{T} 2 \mathrm{~b}$ & 58 & 79 \\
\hline \multicolumn{3}{|l|}{ Tumor grade } \\
\hline Low & 10 & 14 \\
\hline High & 58 & 79 \\
\hline Unknown & 5 & 7 \\
\hline \multicolumn{3}{|l|}{ Size $(\mathrm{cm})$} \\
\hline$\leq$ & 10 & 14 \\
\hline$>5-10$ & 27 & 37 \\
\hline$>10-15$ & 16 & 22 \\
\hline$>15-20$ & 10 & 14 \\
\hline$>20$ & 10 & 14 \\
\hline
\end{tabular}

Abbreviation : MPNST $=$ malignant peripheral nerve sheath tumor.

Other = spindle cell, pleomorphic leiomyosarcoma, mxoi-inflammatory fibroblastic, myxoid, myxoid chondrosarcoma, myofibroblastic, mesenchymal chondrosarcoma, leiomyosarcoma, hemangiopericytoma, fibrosarcoma, fibromyxoid, epithelioid, clear cell, angiosarcoma, and alveolar soft part. 
Table 2

Univariate analysis

\begin{tabular}{|c|c|c|c|c|}
\hline \multirow[b]{2}{*}{ Variable } & \multicolumn{2}{|c|}{ Wound complication frequency } & \multicolumn{2}{|c|}{ Secondary operation frequency orientation="landscape" } \\
\hline & $\% /$ Odds ratio & $\boldsymbol{P}$ & $\% /$ Odds ratio ${ }^{*}$ & $\boldsymbol{P}$ \\
\hline \multicolumn{5}{|l|}{ Categoric } \\
\hline Wound closure by plastic surgery & & .436 & & .093 \\
\hline Yes $(n=46)$ & 28 & & 11 & \\
\hline No $(n=27)$ & 37 & & 26 & \\
\hline Unirradiated vascularized tissue flap & & .559 & & .631 \\
\hline Yes $(n=16)$ & 38 & & 13 & \\
\hline No $(n=57)$ & 30 & & 18 & \\
\hline Gender & & .256 & & .020 \\
\hline Female $(\mathrm{n}=22)$ & 41 & & 32 & \\
\hline Male $(\mathrm{n}=51)$ & 28 & & 10 & \\
\hline Diabetes & & .098 & & .575 \\
\hline Yes $(n=14)$ & 50 & & 21 & \\
\hline No $(n=59)$ & 27 & & 15 & \\
\hline Any history of smoking & & .330 & & .868 \\
\hline Yes $(n=32)$ & 38 & & 16 & \\
\hline No $(n=41)$ & 27 & & 17 & \\
\hline Smoking when diagnosed & & .309 & & .173 \\
\hline Yes $(n=14)$ & 43 & & 29 & \\
\hline No $(n=59)$ & 29 & & 14 & \\
\hline Site & & .056 & & .048 \\
\hline Lower extremity $(\mathrm{n}=49)$ & 39 & & 22 & \\
\hline Upper extremity $(\mathrm{n}=24)$ & 17 & & 4 & \\
\hline Radiation at our institution & & .189 & & .029 \\
\hline Yes $(\mathrm{n}=54)$ & 28 & & 11 & \\
\hline No $(n=18)$ & 44 & & 33 & \\
\hline Dose/fraction (Gy) & & .330 & & .677 \\
\hline $180(\mathrm{n}=42)$ & 33 & & 12 & \\
\hline $200(\mathrm{n}=19)$ & 21 & & 16 & \\
\hline Pathologic grade & & .058 & & .045 \\
\hline $\operatorname{High}(\mathrm{n}=58)$ & 29 & & 14 & \\
\hline Low $(\mathrm{n}=10)$ & 60 & & 40 & \\
\hline Margins & & .552 & & .476 \\
\hline Negative $(n=60)$ & 30 & & 15 & \\
\hline Positive $(\mathrm{n}=13)$ & 38 & & 23 & \\
\hline Chemotherapy before radiation therapy & & .596 & & .092 \\
\hline Yes $(n=12)$ & 25 & & 0 & \\
\hline No $(n=61)$ & 33 & & 20 & \\
\hline Any radiation therapy boost ${ }^{\dagger}$ & & .471 & & .348 \\
\hline
\end{tabular}




\begin{tabular}{|c|c|c|c|c|}
\hline \multirow[b]{2}{*}{ Variable } & \multicolumn{2}{|c|}{ Wound complication frequency } & \multicolumn{2}{|c|}{ Secondary operation frequency orientation="landscape" } \\
\hline & \%/Odds ratio* & $P$ & $\% /$ Odds ratio $*$ & $\boldsymbol{P}$ \\
\hline Yes $(n=13)$ & 23 & & 7 & \\
\hline No $(n=60)$ & 33 & & 18 & \\
\hline Tumor necrosis & & 639 & & .766 \\
\hline $0 \%-50 \%(\mathrm{n}=33)$ & 30 & & 15 & \\
\hline$>50 \%(\mathrm{n}=22)$ & 37 & & 18 & \\
\hline \multicolumn{5}{|l|}{ Continuous } \\
\hline Age at diagnosis & 1.027 & .096 & 1.034 & .108 \\
\hline Maximum tumor dimension & 1.074 & .049 & 1.022 & .613 \\
\hline Radiation dose & 1.000 & .926 & 1.000 & .916 \\
\hline Index surgery date & 1.000 & .196 & 1.000 & .514 \\
\hline Weight (females) & 0.994 & .794 & 1.006 & .820 \\
\hline Weight (males) & 0.989 & .469 & .974 & .188 \\
\hline
\end{tabular}

* Percentages are reported for categoric variables, odds ratios for continuous variables.

${ }^{\dagger}$ Includes intraoperative external beam, postoperative external beam, and catheter-based brachytherapy. 


\section{Table 3}

Summary of wound complications

\begin{tabular}{|lc|}
\hline Complication & $\mathbf{n}$ \\
\hline Secondary operation & \\
Debridement & 8 \\
Debridement with placement of an unirradiated tissue flap & 2 \\
Hematoma evacuation & 2 \\
Outpatient debridement & 4 \\
Outpatient seroma/hematoma evacuation & 3 \\
Readmission for i.v. antibiotics & 2 \\
Deep packing $>120$ d & 2 \\
\hline
\end{tabular}

Abbreviations: i.v. $=$ intravenous. 
Table 4

Factors found to increase risk of a secondary operation on multivariate analysis

\begin{tabular}{|lccc|}
\hline Variable & Parameter estimate & Standard error & $\boldsymbol{P}$ \\
\hline Female gender & 0.96 & 0.40 & .02 \\
Radiation not at our institution & 1.11 & 0.44 & .01 \\
Low grade & 1.17 & 0.50 & .02 \\
\hline
\end{tabular}

Variables listed are only those that were included in the final model. 